\title{
Online Brain Image Repositories for Brain Disease Detection
}

\author{
P. Sriramakrishnan, T. Kalaiselvi, S.T. Padmapriya, S. Ramkumar, K. Ponmozhi
}

\begin{abstract}
Brain image analysis is an emerging area of researchers to improve the diagnosis process more fast and accurate. One of the difficulties is getting the clinical dataset of patients from hospitals to test the performance of the proposed methods. Therefore, numerous online brain image repositories are available to promote the research works. It has manually segmented results to evaluate the accuracy of the developed methods. Each repository has different file format and focused on different problems like skull stripping, tumorous image classification, tumor type categorization, tissue segmentation and tumor with substructure segmentation. This paper gives detail information on famous brain datasets with their purpose.
\end{abstract}

Keywords : Brain repositories, brain datasets, brain disease detection, skull stripping, BraTS datasets, WBA datasets, IBSR datasets

\section{INTRODUCTION}

$\mathrm{H}$ uman brain plays a vital role of the body that controls all other organs. The brain may suffer by several diseases such as Alzheimer, epilepsy, mental disorders, stroke and brain tumors. Physician's takes more time to analyze the brain related diseases compare than other disease by using various imaging modalities such as CT, MRI, and PET. Clinician's cannot give an exact solution to the people without technology. Past few decades, numerous tools and techniques have been developed to serve the health care industry.

The computational methods require a patient data to test their accuracy. In the real time scenario, gathering clinical datasets from the hospital is a tedious process due to privacy of the patients. Recent years, lot of online repositories helps the researchers to do research in health care system. This datasets have manually segmented gold standard results given by the experts to check the accuracy of the proposed methods for researchers.

Revised Manuscript Received on December 16, 2019.

* Correspondence Author

P. Sriramakrishnan*, Department of Computer Applications,

Kalasalingam Academy of Research and Education (Deemed to be University), Krishnankoil, Tamil Nadu, India. sriram0210@gmail.com

T. Kalaiselvi, Department of Computer Science and Applications, The Gandhigram Rural Institute (Deemed to be University), Gandhigram, Tamil Nadu, India kalaiselvi.gri@gmail.com

S.T. Padmapriya, Department of Computer Science and Applications, The Gandhigram Rural Institute (Deemed to be University), Gandhigram, Tamil Nadu, India. stpadmapriya@gmail.com.

S. Ramkumar, Department of Computer Applications, Kalasalingam Academy of Research and Education (Deemed to be University), Krishnankoil, Tamil Nadu, India. ramkumar.dr12013@gmail.com

K. Ponmozhi, Department of Computer Applications, Kalasalingam Academy of Research and Education (Deemed to be University), Krishnankoil, Tamil Nadu, India. chezhiyan71.p@gmail.com
Several human brain online repositories are available on different problems like skull stripping, tumorous image classification, tumor type categorization, tissue segmentation and tumor with substructure segmentation. Famous repositories are BRAINS, BraTS, FIGShare, IBSR, OASIS Brains, and WBA. This paper gives a more details about online brain repositories and their recent methods for comparison.

\section{BRAIN DISEASES}

Each human organ may or may not be affect some of the diseases. Brain also affected by the neurons-degenerative disorders like Alzheimer, Alcoholism, multiple Sclerosis, Schizophrenia, and tumors. Several process in brain images identifies above mention diseases such as skull stripping, tumorous slice detection, brain tissues segmentation, tumor segmentation with substructures, volume rendering and visualization. Several online repositories may helpful to develop the methods for brain disease diagnosis process. This section discuss about the detail information of brain disease.

\section{A. Alzheimer}

Alzheimer disease leads to memory loss and destroys thinking skill. Increasing age is the major factor for this disease. Abnormal deposits of proteins form amyloid plaques and tau tangles throughout the brain. This makes the connection loss between the neurons. Neurons cannot transmit the messages to other parts of the body. This damage initially appears in the hippocampus and entorhinal cortex, which are all essential for forming memories.

\section{B. Alcoholism}

Alcoholism is a type of alcohol addiction disorder which affecting the memory and motivation systems of the brain. Regular alcohol consumption disturbs the balance gamma-aminobutyric acid (GABA) and glutamate in the brain. It has various symptoms like drowsiness, slurred speech, unable to focus, coordination loss, reddish eyes and hangover symptoms. Carbohydrate-deficient transferrin (CDT) test helps to detect the heavy alcohol consumption.

\section{Multiple Sclerosis}

It is a chronic disease that affects the central nervous system especially, brain stem, cerebellum, spinal cord and optic nerves. Immune system attacks myelin sheath that surrounds and protects the nerve fibers, causing inflammation. Myelin also helps the nerves conduct electrical signals quickly and efficiently. This can be categories into four following types such as clinically isolated syndrome, relapse-remitting MS, primary progressive MS, and secondary progressive MS.

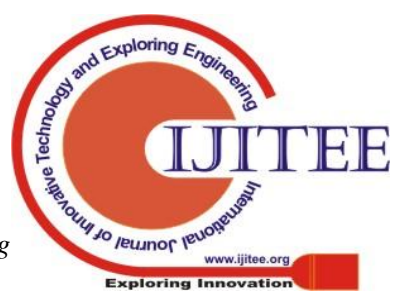




\section{Brain Image Repositories for Brain Disease Detection}

This have following symptoms: muscle weakness, bladder problems, vision and memory problems.

\section{Tumor}

Brain tumor is a collection of mass and uncontrolled abnormal tissue development in the brain. Brain tumor can be benign (non-cancerous) or malignant (cancerous). It can be categorized into two classes namely primary and secondary. Primary brain tumor originates from brain whereas secondary starts elsewhere in the body and spreads to the brain. Primary brain tumor may be either benign or malignant whereas secondary are all malignant. There are more than 120 types of brain tumors categorized by the world health organization (WHO). Various categories of brain tumors operated by the National Institute of Mental Health and Neurosciences (NIMHANS), Bangalore, India, during January 2010 to December 2014 on Indian patients. The most affected tumor reported by NIMHANS data is meningioma $20 \%$, followed by glioblastoma $14 \%$. Meningioma is a benign tumor that arises from the meninges and surgeons can remove completely. Glioblastomas are a subset of gliomas that arise from astrocytes, or the star-shaped cells in the brain. These are highly malignant because the cells reproduce quickly and reduce the survival rate of the patient. The substructures in a brain tumor are edema, necrosis, non-enhancing core and active core or enhancing tumor.

\section{ONLINE BRAIN REPOSITORIES}

Online brain repositories are very important to develop the novel method in all the problems. It can be helpful in train and test the algorithm using machine learning algorithms. There are lot of problems identified in the brain image processing such as skull stripping, stroke identification, tumor segmentation with substructures, tumor image classification, tumor type identification and Alzheimer diseases detection. This section describes the following state-of-an-art human brain repositories available in online.

\section{A. BRAINS}

Brain Images of Normal Subjects (BRAINS) repository is designed for normal individuals [1]. This datasets can useful in the sense of measuring pressure of blood, body mass index (BMI), cognitive ability and detecting abnormality. This datasets contains 808 normal datasets and collected from the age of 15 to 81 [2]. The datasets holds the T1, T2, T2* and FLAIR images in DICOM format.

\section{B. BraTS}

Multimodal Brain Tumor Segmentation Challenge (BraTS) repository evaluates proposed methods accuracy with the dataset given by the expert team [3]. The updated BraTS datasets have tumor with sub structure annotated labels for complete tumor, core tumor, and enhancing tumor. BraTS datasets have high grade Glioma (HGG) and low grade Glioma (LGG) tumor. The image dimension is $240 \times 240 \times 155$. All datasets are re-sampled to $1 \times 1 \times 1 \mathrm{~mm}$ and skull stripped [4].

BraTS datasets split into three types such as training data, challenge data and leader board. Training datasets helps to test the methods with manually segmented results for training purpose. Remaining two types are helps to evaluate the accuracy of the method to several existing methods. The dataset is produced with different versions, in the year 2013, 2015 and 2018. BraTS2013 contains 30 patient sets including $20 \mathrm{HGG}$ and 10 LGG [6]. BraTS2015 and BraTS2018 training datasets contains 285 cases included 210 HGG and 75 LGG [5]. Some existing results on BraTS is given in Table 1. This dataset also helps to develop the methods in tumor image detection.

\section{FIGShare}

FIGShare dataset contains three different types of 3064 contrast enhanced T1 brain tumor images [7]. These images are collected from 233 patients and 708 images for meningioma, 1426 images for glioma, 930 images for pituitary tumor [8]. The images have the $512 \times 512$ image size, thickness of slice is around $6 \mathrm{~mm}$ and the interslice gap is 1 $\mathrm{mm}$. This dataset is very useful to train the algorithm using the images for tumor type categorization. State of the art methods results on FiGshare dataset is shown in Table 2 [9].

Table 2: Existing results of FIGShare dataset

\begin{tabular}{|l|l|l|l|}
\hline Work & Method & Accuracy & $\begin{array}{l}\text { Training } \\
\text { data }\end{array}$ \\
\hline $\begin{array}{l}\text { Jun } \\
\text { Cheng }\end{array}$ & BoW-SVM & $91.28 \%$ & $80 \%$ \\
\hline Ismael & DWT-Gabor-NN & $91.90 \%$ & $70 \%$ \\
\hline Pashaei & CNN-ELM & $93.68 \%$ & $70 \%$ \\
\hline Nyoman & CNN & $84.19 \%$ & - \\
\hline Afshar & CapsNet & $90.89 \%$ & - \\
\hline Deepak & deep CNN-SVM & $97.1 \%$ & $56 \%$ \\
\hline
\end{tabular}

\section{IBSR}

IBSR provides manually segmented results with MR images. Varieties of datasets are available based on the problems identified in the brain image processing. The repository is concentrate skull stripping, brain tissue segmentation of normal subject, and tumor segmentation. It is more famous for skull stripping and brain tissue segmentation.

There are two type of dataset were frequently used under IBSR. The dataset are IBSR20 and IBSR18 from the IBSR with gold standard [10]. The first dataset is a collection of 20 subject's T1-weighted coronal scans. The image size is $256 \times$ 256 with around 60 images with $1 \mathrm{~mm} \times 1 \mathrm{~mm} \times 3 \mathrm{~mm}$ voxel resolution. IBSR 18 is composed by $18 \mathrm{~T} 1-\mathrm{w}$ scans with $256 \times$ $256 \times 128$ and $1.5 \mathrm{~mm}$ thickness. The dataset is also supplied with their gold standard which contains the manually labeled volumes into three-classes are CSF, GM and WM tissue.

Skull stripping or brain portion extraction is a preprocessing step for tumor segmentation, registration and visualization and tissue segmentation. Removal of non brain portion is a important task to increase the accuracy of prognostic procedures. Table 3 shows the results of the existing methods on skull stripping on IBSR20 [11]. Likewise tissue segmentation is more important to identified many neuro-disorder like tumor, stroke, and Alzheimer dieses. Develop a method for segmenting brain tissues into GM, $\mathrm{WM}$, and cerebrospinal fluid CSF is more reduce the manually segmentation time for the physicians. Table 4 shows that the existing methods on IBSR20 dataset for brain 
tissue segmentation.

Table 1: Existing methods results on BraTS2013

\begin{tabular}{|c|l|cc|c|}
\hline S. No & Author's & \multicolumn{2}{|c|}{$\begin{array}{c}\text { Dice Avg. } \\
\text { HG/LG }\end{array}$} & $\begin{array}{l}\text { Cmputing } \\
\text { Time (mins) }\end{array}$ \\
\hline 1 & Bauer et al., (2013) & 68 & $74 / 49$ & 8 \\
\hline 2 & Buendia et al., (2013) & 57 & $71 / 19$ & 0.3 \\
\hline 3 & Cordier et al.,(2013) & 68 & $71 / 60$ & 20 \\
\hline 4 & Demirhan et al. (2015) & 61 & NA/NA & 0.4 \\
\hline 5 & Doyle (2013) & 74 & $78 / 63$ & 15 \\
\hline 6 & Festa et al., (2013) & 62 & $77 / 24$ & 30 \\
\hline 7 & Geremia et al., (2012) & 62 & $65 / 55$ & 10 \\
\hline 8 & Guo (2013) & 74 & $75 / 71$ & $<1$ \\
\hline 9 & Hamamci (2012) & 72 & $78 / 55$ & 20 \\
\hline 10 & Meier (2014) & 69 & $77 / 46$ & 6 \\
\hline 11 & Reza (2013) & 70 & $77 / 52$ & 90 \\
\hline 12 & Raviv (2012) & 74 & $74 / \mathrm{NA}$ & 8 \\
\hline 13 & Shin (2012) & 75 & $81 / 28$ & 8 \\
\hline 14 & Subbanna (2013) & 44 & $51 / 24$ & 70 \\
\hline 15 & Taylor et.al., (2013) & 75 & $78 / 68$ & 100 \\
\hline 16 & Tustison (2013) & 76 & $79 / 67$ & 20 \\
\hline 17 & Zhao (2013) & 75 & $80 / 62$ & 2 \\
\hline 18 & Zikic (2012) & \multicolumn{2}{|c|}{} \\
\hline
\end{tabular}

Table 3: Skull stripping results of state-of-an-art methods on IBSR20

\begin{tabular}{|l|l|l|l|l|l|}
\hline Method & \multicolumn{1}{|c|}{ Dice Similarity } & \multicolumn{1}{|c|}{ Sensitivity } & \multicolumn{1}{c|}{ Specificity } & FPR & \multicolumn{1}{c|}{ FNR } \\
\hline BET & $0.7417 \pm 0.146$ & $0.9987 \pm 0.002$ & $0.9157 \pm 0.055$ & 0.878 & 0.003 \\
\hline BSE & $0.8747 \pm 0.088$ & $0.8857 \pm 0.116$ & $0.997 \pm 0.012$ & 0.086 & 0.115 \\
\hline MLS & $0.9057 \pm 0.213$ & $0.9367 \pm 0.22$ & $0.9877 \pm 0.017$ & 0.119 & 0.064 \\
\hline BEM2D & $0.9137 \pm 0.033$ & $0.9097 \pm 0.063$ & $0.9927 \pm 0.006$ & 0.066 & 0.091 \\
\hline $\begin{array}{l}\text { BEM2D } \\
\text { E }\end{array}$ & $0.9387 \pm 0.021$ & $0.9517 \pm 0.036$ & $0.9927 \pm 0.006$ & 0.075 & 0.049 \\
\hline
\end{tabular}

Table 4: Existing methods on IBSR20 for brain tissue segmentation

\begin{tabular}{|l|c|c|c|}
\hline \multicolumn{1}{|c|}{ Name } & CSF & GM & WM \\
\hline KM & $0.13 \pm 0.10$ & $0.67 \pm 0.14$ & $0.78 \pm 0.05$ \\
\hline FCM & $0.27 \pm 0.16$ & $0.78 \pm 0.07$ & $0.74 \pm 0.04$ \\
\hline FFCM & $0.11 \pm 0.03$ & $0.69 \pm 0.03$ & $0.80 \pm 0.04$ \\
\hline FAST & $0.13 \pm 0.04$ & $0.68 \pm 0.06$ & $0.79 \pm 0.10$ \\
\hline SPM5 & $0.17 \pm 0.07$ & $0.76 \pm 0.06$ & $0.80 \pm 0.04$ \\
\hline SPM8 & $0.21 \pm 0.07$ & $0.78 \pm 0.06$ & $0.81 \pm 0.08$ \\
\hline GAMIXTURE & $0.25 \pm 0.12$ & $0.77 \pm 0.09$ & $0.74 \pm 0.02$ \\
\hline ANN & $0.15 \pm 0.06$ & $0.69 \pm 0.09$ & $0.77 \pm 0.14$ \\
\hline
\end{tabular}

Table 5: Results of tumorous slice detection of existing methods in WBA

\begin{tabular}{|l|l|l|l|l|l|l|}
\hline No. & Method Name & FA \% & MA \% & Sensitivity & Specificity & Accuracy \\
\hline 1 & PCB & 15.1 & 26 & 84.8 & 74.0 & 58.8 \\
\hline 2 & Avinash Method & 6.6 & 6.1 & 93.4 & 93.9 & 87.3 \\
\hline 3 & Hybrid Features & 7.6 & 4.1 & 92.4 & 95.9 & 88.3 \\
\hline 4 & Wavelet & 5.3 & 5.1 & 94.7 & 94.9 & 89.6 \\
\hline
\end{tabular}

\section{E. WBA}

WBA contains eight datasets which includes two normal $\left(\mathrm{V}_{1} \& \mathrm{~V}_{2}\right)$ and six tumorous datasets $\left(\mathrm{V}_{3}-\mathrm{V}_{8}\right)$ [12]. Each dataset has around sixty slices with $256 \times 256$ pixels with thickness of slice varying from 2 to $5 \mathrm{~mm}$. Identifying tumorous slices is an important process which saves the time and improves the accuracy. Normally MRI volume has both normal and tumorous image. Machine learning algorithms help to separate the image into either normal or tumorous image by using SVM, random forest, and naive bias. Table 5 shows the tumorous slice classification results of state-of-the-art method on WBA datasets [13].

\section{CONCLUSION}

This paper gives a brief knowledge about available online brain image repositories. In addition, importance of each repository also discussed with imaging parameters, resolution, inter-slice gap, field of view, and slice thickness. Developed state-of-an-art methods on each repository also briefly discussed with

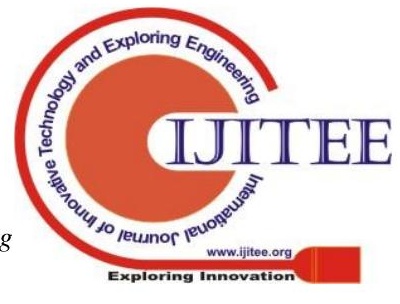




\section{Brain Image Repositories for Brain Disease Detection}

suitable evaluation metrics.

\section{REFERENCES}

1. Job, D.E., Dickie, D.A., Rodriguez, D., Robson, A., Danso, S., Pernet, C., Bastin, M.E., Boardman, J.P., Murray, A.D., Ahearn, T. and Waiter, G.D., 2017. A brain imaging repository of normal structural MRI across the life course: Brain Images of Normal Subjects (BRAINS). NeuroImage, 144, pp.299-304.

2. BRAINS datasets, https://datashare.is.ed.ac.uk/handle/10283/720

3. BraTS2013 datasets https://www.smir.ch/BRATS/Start2013

4. Myronenko, A., 2018, September. 3D MRI brain tumor segmentation using autoencoder regularization. In International MICCAI Brainlesion Workshop (pp. 311-320). Springer, Cham.

5. Menze, B.H., Jakab, A., Bauer, S., Kalpathy-Cramer, J., Farahani, K. Kirby, J., Burren, Y., Porz, N., Slotboom, J., Wiest, R. and Lanczi, L. 2014. The multimodal brain tumor image segmentation benchmark (BRATS). IEEE transactions on medical imaging, 34(10), pp.1993-2024.

6. BraTS2015 datasets https://www.smir.ch/BRATS/Start2015

7. FIGShare Dataset, https://figshare.com/articles/brain_tumor_dataset/1512427/5

8. Cheng, J., Huang, W., Cao, S., Yang, R., Yang, W., Yun, Z., Wang, Z. and Feng, Q., 2015. Enhanced performance of brain tumor classification via tumor region augmentation and partition. PloS one, 10(10), p.e0140381

9. S.Deepak and P.M.Ameer, Brain Tumor Classification Using Deep CNN Features via Transfer Learning, Computers in Biology and Medicine, Volume 111, August 2019, 103345

10. Internet Brain Segmentation Repository, Center for Morphometric Analysis at Massachusetts General Hospital, dataset available at: http://www.cma.mgh.harvard.edu/ibsr/index.html.

11. Somasundaram, K. and Kalaiselvi, T., 2011. Automatic brain extraction methods for $\mathrm{T} 1$ magnetic resonance images using region labeling and morphological operations. Computers in biology and medicine, 41(8), pp.716-725.

12. IBSR Dataset, http://www.med.harvard.edu/AANLIB/home.html

13. Kalaiselvi, T., Sriramakrishnan, P. and Somasundaram, K., 2016 December. Brain abnormality detection from MRI of human head scans using the bilateral symmetry property and histogram similarity measures. In 2016 International Computer Science and Engineering Conference (ICSEC) (pp. 1-6). IEEE. 